\title{
Modelling of solidification of binary fluids with non-linear viscosity models
}

\author{
T. Wacławczyk, D. Sternel \& M. Schäfer \\ Department of Numerical Methods in Mechanical Engineering, \\ Technische Universität Darmstadt, Germany
}

\begin{abstract}
This paper deals with the numerical modelling of multiphase flows with phase transition during solidification of binary alloys. First, verification of the effective viscosity assumption in the regime of moving solid (equiaxed crystals) and liquid (melt) for large solid mass fractions is presented. In order to extend the effective viscosity model to the region of stationary solid (columnar crystals); non-linear dependence of the viscosity on the solid mass fraction and the shear velocity is introduced based on the experimental evidence. The proposed formulation is used in a numerical study of the metal alloy solidification in a rectangular cavity. Keywords: multiphase flow, solidification, mushy zone, phase change.
\end{abstract}

\section{Introduction}

During solidification of binary fluids, e.g. metal alloy $\mathrm{Al}-\mathrm{Si}, \mathrm{Al}-\mathrm{Cu}$, for a certain range of temperatures and compositions a mushy zone is created (cf. Refs. [1,2]). The influence of the mushy zone morphology on the flow field can be modelled by two approaches: porous media model, where the Carman-Kozeny relation couples local porosity of the medium with the local liquid fraction, or direct modification of the local fluid viscosity relatively to the local solid fraction, see e.g. Refs. [3,4], respectively. These two physical models are valid in different regions of the mushy layer: the region of stationary, columnar crystals where the solid velocity is $\vec{u}_{s}=0$ and the region of the equiaxed crystals where the velocity of solid is assumed to be equal to the velocity of the melt $\vec{u}_{s}=\vec{u}_{l}$, see Fig. 1 . The difficulties in the modelling of the mushy zone by a one-field model arise during the transition from the mixture velocity (for fluid and solid) to the interstitial velocity in the porous zone, see Refs. $[5,6]$. 


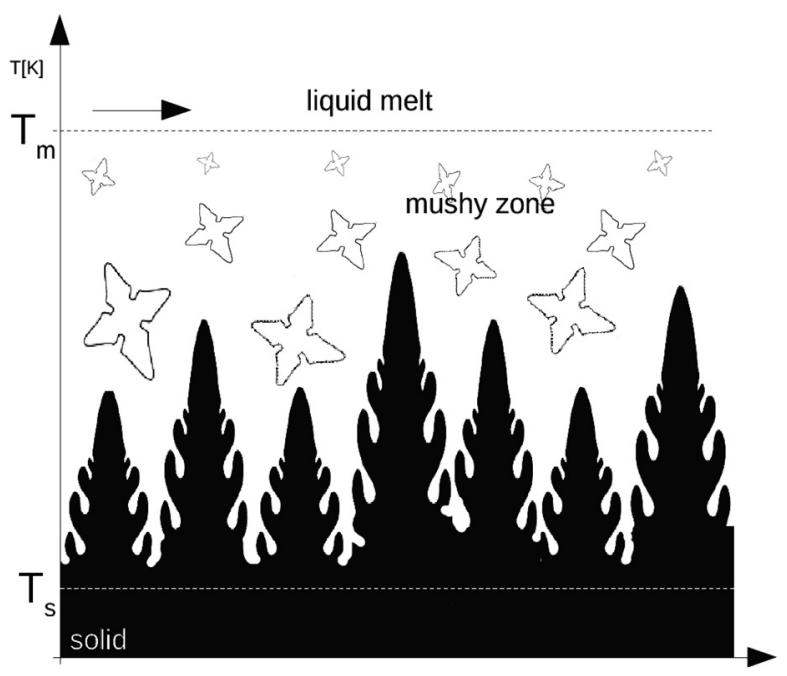

Figure 1: Schematic presentation of the mushy zone with two characteristic regions: columnar dendrites $\vec{u}_{s}=0$ and advected equiaxed crystals $\vec{u}_{s} \approx \vec{u}_{l}$.

The disadvantage of the first method is the necessity of the permeability coefficient estimation by experimental or theoretical investigations. This issue is not straightforward because the mushy zone has a complex morphology dependent on the material and external conditions. A porous media model should be used only in the region where the solid phase is stationary.

In the case of the second approach, an assumption about the continuous change of the material properties across the solid, the mushy layer and the liquid is used. A common approach employs a lever rule to approximate density and viscosity in the mushy zone together with a linear dependence of the solid fraction on temperature inherited from the linearised phase change diagram, see Fig. 2 and Refs. [5, 6]. The linear dependence of solid and liquid viscosities based on the lever rule is not appropriate since the solid viscosity $\mu_{s}$ can not be defined. An alternative for the linear viscosity approximation was given in Ref. [7], however, it does not take into account the dependence of the viscosity on the shear velocity.

In this paper, based on the experimental evidence from Ref. [8] and the parametrisation study from Ref. [9], we postulate an alternative non-linear dependence of the viscosity on the solid fraction and the shear velocity. The viscosity values used for partial validation of the model were obtained during measurements in a cylindrical rheometer, see Ref. [8]. In section 3 experimentally obtained data, i.e. viscosities as a function of solid fraction and the shear velocity, were used to confirm the possibility of a modelling of multiphase systems by the effective viscosity model.

Numerical simulations presented in this paper were carried out with the commercial software Star-CD where the user coding was used for implementation of 
the physical models. The proposed viscosity data parametrisation is tested in the case of the binary alloy solidification in a rectangular cavity, see Ref. [3].

\section{Description of the solidification model}

A solidification model implemented in the Star-CD (ver. 4.06) commercial software is employed. The description of the model is given in supplementary notes distributed together with the program, for this reason here only a short comparison of the model used and other models presented in the literature, see Refs. $[1,3,5]$, is given.

The set of the conservation equations that describe the mixture medium is obtained by the volume averaging under additional assumptions: equal solid/liquid velocity $\vec{u}_{s}=\vec{u}_{l}$, equal solid/liquid density $\rho_{s}=\rho_{l}=\rho$ and the mechanical equilibrium $p_{s}=p_{l}=p$. The set of conservation equations consists of: the momentum, the continuity, the energy and the species mass fraction transport equations:

$$
\begin{gathered}
\frac{\partial\left(\rho u_{i}\right)}{\partial t}+\frac{\partial\left(\rho u_{j} u_{i}\right)}{\partial x_{j}}=-\frac{\partial p}{\partial x_{i}}+\frac{\partial}{\partial x_{j}}\left[\mu\left(\frac{\partial u_{i}}{\partial x_{j}}+\frac{\partial u_{j}}{\partial x_{i}}\right)-\frac{2}{3} \mu \frac{\partial u_{l}}{\partial x_{l}} \delta_{i j}\right] \\
+\rho_{\text {ref }} g_{i}\left[1-\beta\left(T-T_{r e f}\right)\right], \\
\frac{\partial \rho}{\partial t}+\frac{\partial\left(\rho u_{j}\right)}{\partial x_{j}}=0, \\
\frac{\partial(\rho h)}{\partial t}+\frac{\partial\left(\rho u_{j} h\right)}{\partial x_{j}}=\frac{\partial p}{\partial t}+u_{j} \frac{\partial p}{\partial x_{j}}+\tau_{i j} \frac{\partial u_{i}}{\partial x_{j}}+k \frac{\partial^{2} T}{\partial x_{j}^{2}}, \\
\frac{\partial(\rho C)}{\partial t}+\frac{\partial\left(\rho u_{j} C\right)}{\partial x_{j}}=D \frac{\partial^{2} C}{\partial x_{j}^{2}} .
\end{gathered}
$$

One can notice that the mathematical model in the Star-CD allows for simulation of the material compressibility, therefore, modelling of the shrinkage effects is possible. In the mushy zone the variables and the material properties in the above equations represent quantities obtained by volume averaging (see Ref. [5,6]). Hence, the velocity $u_{i}$, the density $\rho$, the viscosity $\mu$, the enthalpy $h$, the thermal conductivity $k$, the species mass fraction $C$ and the species diffusion coefficient $D$ represent mass averaged quantities of the solidified alloy (solid) and the melt (fluid) mixture:

$$
\phi=C_{s} \phi+C_{l} \phi
$$

where $C_{s}=\rho_{s} c_{s} / \rho$ and $C_{l}=\rho_{l} c_{l} / \rho$ are solid and liquid mass fractions, $c_{s}$ and $c_{l}$ denote solid and liquid volume fractions and $\phi$ represents the aforementioned variables and the material properties of the mixture. The local thermodynamic equilibrium assumption allows to define the temperature $T$ as the equilibrium 


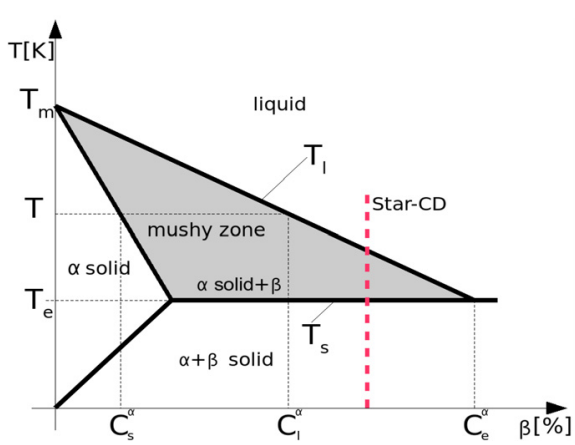

a)

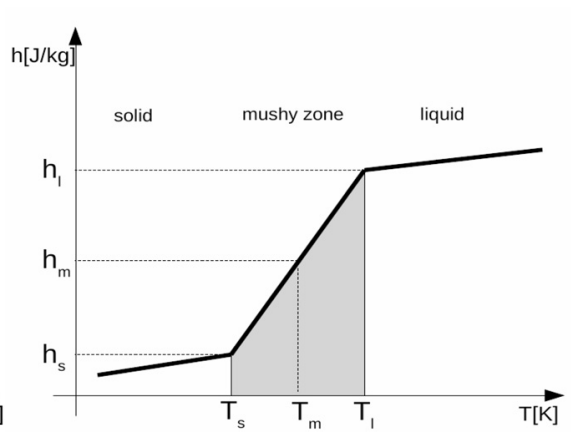

b)

Figure 2: The phase change diagram a) typical for binary fluids of $\alpha+\beta$ composition, b) approximation of the phase diagram currently used in the Star-CD valid for the constant melt composition.

temperature. Therefore the buoyancy effects can be approximated using the Boussinesq assumption, see Eq. (1). The enthalpy in the solid, liquid and mushy zone are calculated as follows, see Fig. 2b:

$$
\begin{gathered}
h_{s}=\left\{\begin{array}{cc}
c_{p s} T: & T \leq T_{s} \\
c_{p s} T_{s}: & T_{s}<T<T_{l}
\end{array}\right. \\
h_{l}=\left\{\begin{array}{c}
c_{p l} T+\left(c_{p s}-c_{p l}\right) T_{m}+L \quad: \quad T \geq T_{l} \\
c_{p l} T_{l}+\left(c_{p s}-c_{p l}\right) T_{m}+L \quad: \quad T_{s}<T<T_{l}
\end{array}\right.
\end{gathered}
$$

where $L$ is the latent heat of fusion, $c_{p l}, c_{p s}$ are specific heats of the liquid and solid, respectively, and $T_{m}=\left(T_{s}+T_{l}\right) / 2$ where $T_{s}, T_{l}$ are solidus and liquidus temperatures.

The Star-CD code uses a simplified phase change diagram where the enthalpy is calculated for constant (initial) composition of the binary fluid, see Fig. 2b. This kind of simplification is the source of the main difference between solidification model known in literature and the procedure applied in Star-CD. The equation used for the determination of the liquid volume fraction $c_{l}$ is deduced from the simplified phase change diagram, cf. Fig. 2b:

$$
c_{l}=\frac{T-T_{s}}{T_{l}-T_{s}}, \quad c_{s}=1-c_{l},
$$

where solidus $T_{S}$ and liquidus $T_{l}$ temperatures are constant and must be supplied by the user, whereas the calculation of the liquid mass fractions from the phase change diagram in Fig. 2a requires information about the local composition $C$ 
since it influences its solidus and liquidus temperatures, see Refs. [5, 10]:

$$
C_{l}=\frac{C-C_{s}^{\alpha}}{C_{l}^{\alpha}-C_{s}^{\alpha}}, \quad C_{l}=\frac{\rho_{l} c_{l}}{\rho} .
$$

The approximation in Star-CD is based on the assumption that the composition of the binary fluid remains constant. The main consequence of this simplification is a direct dependence of the liquid volume fraction distribution on the temperature since $T_{s}, T_{l}$ are set constant in Eq. (8). Hence, one can expect that according to Eq. (8), the $c_{l}$ distribution must follow isotherms. Thus, modelling of the real shape of the solidification front is largely an approximation. One can also notice another implication of the simplified model. The conservation equation (4) is no more a species mass conservation equation but only a solid and liquid mass conservation equation since the composition of the binary liquid is constant.

\section{Verification of the effective viscosity model $C_{s} \leq \mathbf{0 . 4 5}$}

To verify the hypothesis about the applicability of the effective viscosity model in the case of large solid fractions, experimental data obtained from viscosity measurements of a $A l-S i$ metal alloy carried out in Ref. [8] were used. During the experiment, a cylindrical rheometer was placed in an electrical thermostat allowing to sustain constant temperature around it. Measurements of the torque $M$ on the grooved rod rotating inside the cylinder allowed to calculate value of the tension acting at the surface of the rod, see Eq. (10). The number of the revolutions per minute $n$ was used to calculate the shear velocity $\gamma$, cf. Eq. (11). The ratio of the tension $\tau$ and the shear velocity $\gamma$ gives the viscosity of the multiphase fluid, see Eq. (12):

$$
\begin{gathered}
\tau=\left(\frac{1}{2 \pi L R_{i}^{2} C_{L}} \frac{1+\delta^{2}}{2 \delta^{2}}\right) M, \\
\gamma=\left(\frac{1+\delta^{2}}{\delta^{2}-1} \frac{\pi}{30}\right) n, \\
\mu=\frac{\tau}{\gamma},
\end{gathered}
$$

where $\delta=R_{a} / R_{i}$ is the ratio of the cylinder radius $R_{a}$ and the rod radius $R_{i}$. It is important to notice that Eqs. (10-12) are valid only under the assumption of a linear velocity profile between the external cylinder surface and the rotating rod, i.e. a Couette flow assumption.

Since the whole cylindrical rheometer was placed inside of the thermostat, it was possible to assume that the temperature and thus the solid mass fraction are constant during the simulation. This simplification allows to employ only the momentum and continuity equations, see Eqs. (1-2) respectively, where all variables and material properties are defined for the multiphase mixture. The isothermal assumption allow for relatively straightforward simulation of this 
multiphase flow. The single simulation point in Fig. 4 (left) corresponds to the single viscosity measurement for given shear velocity $\gamma$ see Eq. (11). Experimental data for two temperatures $594^{\circ} \mathrm{C}, 605^{\circ} \mathrm{C}$ were used, density $\rho=2700 \mathrm{~kg} / \mathrm{m}^{3}$ was assumed constant during all simulations. After the convergence criterion for the torque calculated on the grooved rod is obtained (variation of the $z$-component of the total torque was monitored) the new values of the tension, see Eq. (10) and then viscosity, see Eq. (12), are calculated and compared with experimental findings, see Fig. 4. Obtained results show that when using exact experimental data, the flow in the multiphase system is accurately modelled with the effective viscosity assumption. The difference between the experimental value and the numerical solution $\epsilon=1-\mu_{c f d} / \mu_{\text {exp }}$ can be defined due to the knowledge about the measured viscosities $\mu_{\text {exp }}$. One can notice that the value of $\epsilon$ grows with increasing shear velocity $\gamma$, see Fig. 4 (left). In the case of the solid mass fraction $C_{s} \approx 45 \%$, the error $\epsilon$ starts to grow from the value $\epsilon=3.9 \%, \gamma \approx 4901 / \mathrm{s}$ until $\epsilon \approx 22 \%$ for $\gamma \approx 5201 / \mathrm{s}$ the last computational point in Fig. 4 (left, top). For the solid mass fraction $C_{s}=33 \%$ the error $\epsilon$ grows from $\epsilon \approx 3.9 \%$, $\gamma \approx 5001 / \mathrm{s}$ until the solution with the experimentally obtained viscosities does no more follow the experimental data, i.e., $\epsilon \approx 44 \%, \gamma \approx 6001 / s$. The source of the error variation has a twofold nature. First of all, in the case of large solid fractions, for larger shear velocities $\gamma$ the non-slip condition at the wall of the cylindrical rheometer and the wall of the grooved rod (rod is grooved to avoid slip effect) can be no more satisfied. Secondly, the important factor that limits the range of the measurements in the cylindrical rheometer is the development of the Taylor instability. This phenomenon occurs for large values of the revolutions per minute

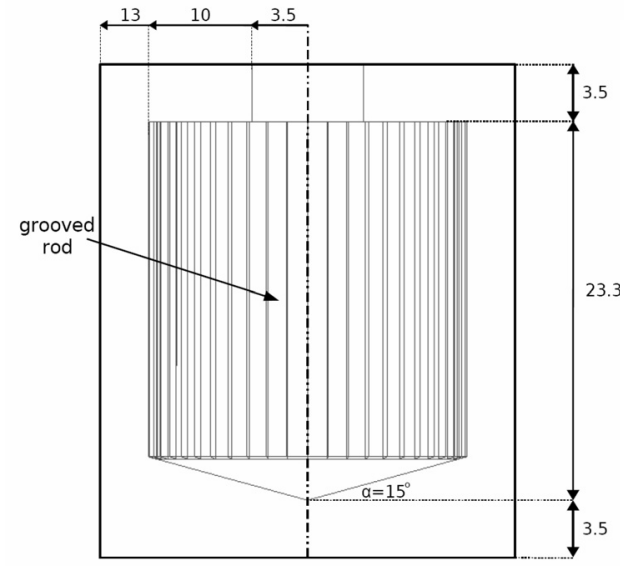

a)

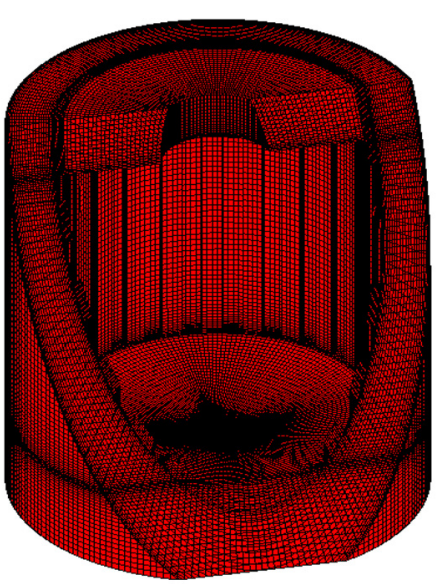

b)

Figure 3: The cylindrical rheometer a) characteristic dimensions of the grooved rod and the cylinder $R_{a}=13 \mathrm{~mm}, R_{i}=10 \mathrm{~mm}$, b) cross section through the numerical model build from about $8 \times 10^{5} \mathrm{CV}$ 's. 

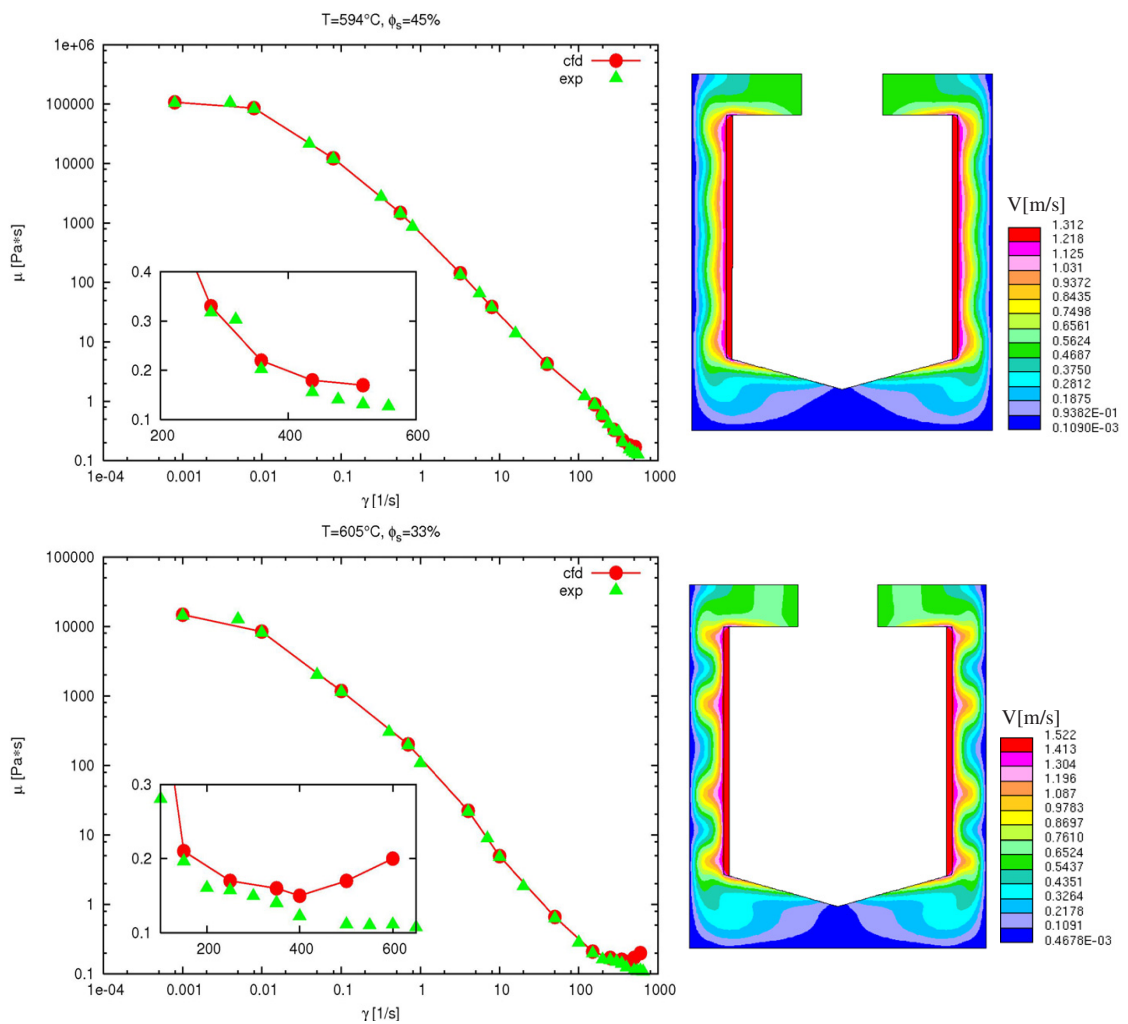

Figure 4: The viscosity obtained from simulations in the cylindrical rheometer compared with experimental data (left) and velocity magnitude for the last computational point (right) in the case of the two solid mass fractions $C_{s}=45 \%$ (top), $C_{s}=33 \%$ (bottom). In the case of smaller solid mass fraction $C_{s}=33 \%$ vortices developed due to the Taylor instability are visible.

$n$ and prevents accurate viscosity measurements since the Couette flow assumption is no more satisfied, see Fig. 4. The accurate prediction of this effect in multiphase systems is difficult, since it is directly connected with the variable viscosity of the mixture fluid. The numerical modelling of the flow in the cylindrical rheometer should be further investigated since it might become a valuable verification tool for the experimental investigations.

\section{Extension of the viscosity model for $C_{s}>0.45$}

The key problem during modelling of the mushy zone by the effective viscosity model is the extension of this assumption for large solid fractions $C_{s}>0.45$ since in this case viscosity measurements in the rheometer are not possible. In fact, 


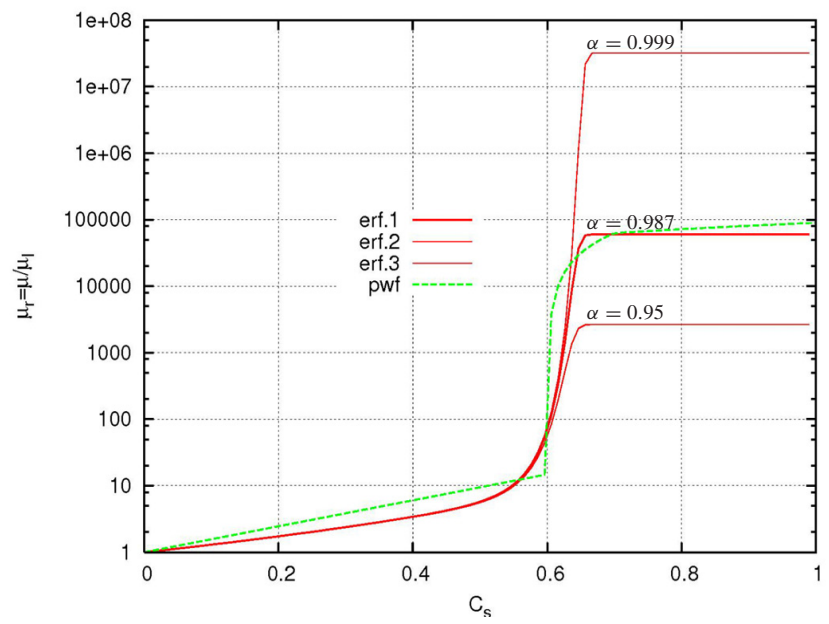

Figure 5: Two parametrisations of the effective viscosity: error function erf and piecewise functional $p w f$ viscosity parametrisations. In the case of the erf parametrisation three different $\alpha$ values were used $0.95,0.987,0.999$, coefficients $\beta=1 e-4, \gamma=9.81$ are the same for all three cases. The second $p w f$ parametrisation uses $\mu_{l}=1 \mathrm{~Pa} \cdot s, \mu_{s}=9 e+4 \mathrm{~Pa} \cdot s$ and $C_{s, c r}=0.6$. Notice that the relative viscosity erf parametrisation $\mu_{r}=\mu / \mu_{l}$ is almost independent of the $\alpha$ value when $C_{s} \leq 0.5$.

viscosity of the solid $\mu_{s}$ can be considered only as an auxiliary parameter that does not possess physical meaning. For this reason the commonly used approximation:

$$
\mu=C_{s} \mu_{s}+\left(1-C_{s}\right) \mu_{l},
$$

where $\mu_{l}$ is the liquid viscosity, is not valid since $\mu_{s}$ can be an arbitrarily large number. Alternatives for this approach are rarely presented in the literature, two examples given in Refs. [4,9] will be shortly discussed below.

In the case of the first parametrisation, the mixture viscosity $\mu$ is approximated by a piecewise functional approach, cf. Fig. 5:

$$
\mu\left(C_{s}\right)=\left\{\begin{array}{ccc}
\mu_{l} e^{4.5 C_{s}} & : \quad C_{s} \leq C_{s, c r} \\
b_{1} C_{s}+b_{2} & : C_{s, c r}+0.1>C_{s}>C_{s, c r} \\
\left(1-C_{s}\right) \mu_{l}+C_{s} \mu_{s}: & C_{s} \geq C_{s, c r}+0.1
\end{array}\right.
$$

where $C_{s, c r}$ (here after Ref. [4] $C_{s, c r}=0.6$ ) is the critical solid fraction value that defines the highly viscous zone interpreted as the columnar crystals region, see Fig. $1 ; b_{1}$ and $b_{2}$ are two constants determined by the solution of the two equation system in point $C_{s, c r}, C_{s, c r}+0.1$. One needs to notice that Eq. (14) employs also the linear dependence given by equation Eq. (13). The main disadvantage of this approach is its discontinuity, cf. Fig. 5, and its lack of physical justification. 
The second effective viscosity parametrisation uses error function to approximate variation of the effective viscosity $\mu\left(C_{S}\right)$ :

$$
\mu\left(C_{S}\right)=\mu_{l}\left\{1-\alpha \cdot \operatorname{erf}\left(\frac{\sqrt{\pi}}{2} C_{s}\left[1+\frac{\beta}{\left(1-C_{S}\right)^{\gamma}}\right]\right)\right\}^{-B / \alpha}
$$

where $\alpha, \beta, \gamma$ are parameters to set, $B=2.5$, cf. Ref. [9]. The second approach is non-linear and the error function is known to be a solution of the heat transport equation, when the initial condition is given by the Heaviside function. In the case of solidification in the mushy zone, the aforementioned condition can be interpreted as the jump of the enthalpy caused by the latent heat rejected from the solid to the liquid phase, cf. Fig. 2b. Since the solid fraction variation across the mushy zone is expressed by this function, see Ref. [1], and the effective viscosity depends on the solid fraction, it should also be possible to express it in terms of the error function.

\subsection{Dependence on the shear velocity $\gamma$}

To obtain a formula for the effective viscosity $\mu$ as a function of the solid fraction $C_{s}$ and the shear velocity $\gamma$, additional normalised variables are introduced: the normalised viscosity $\mu_{r}=\mu / \mu_{l}$ and the normalised shear velocity $\gamma_{r}=\gamma / \gamma_{C}$. The values of $\mu_{l}, \mu_{s}$ and $\gamma_{C}$ were set to $0.02 \mathrm{~Pa} \cdot s, 500 \mathrm{~Pa} \cdot s$ and $10001 / \mathrm{s}$, respectively, based on the available experimental data for five temperatures $T$ : $594^{\circ} \mathrm{C}, 600^{\circ} \mathrm{C}, 605^{\circ} \mathrm{C}, 610^{\circ} \mathrm{C}, 615^{\circ} \mathrm{C}$ and corresponding solid fractions $C_{s}: 0.45$, $0.39,0.33,0.25,0.17$, see Fig. 6 . First, fitting of the continuous functions to the normalised viscosities $\mu_{r}\left(\gamma_{r}\right)$ obtained for each solid fraction was carried out, in order to obtain $\mu_{r}\left(\gamma_{r}, C_{s}=\right.$ const . $)$. Afterwards, the obtained functions were used to calculate formula for the $B$ coefficient. The remaining coefficients $\alpha=0.988$, $\beta=1 e-4, \gamma=9.66$ were set only once and are constant in the whole domain. The new, effective viscosity parametrisation $\mu_{r}\left(C_{s}, \gamma_{r}\right)$ is given by the following equations:

$$
\begin{gathered}
\mu_{r}\left(C_{s}, \gamma_{r}\right)=\left\{1-\alpha \cdot \operatorname{erf}\left(\frac{\sqrt{\pi}}{2} C_{s}\left[1+\frac{\beta}{\left(1-C_{S}\right)^{\gamma}}\right]\right)\right\}^{-B\left(\gamma_{r}\right) / \alpha}, \\
B=\dot{\gamma}^{-0.52}+1.1 .
\end{gathered}
$$



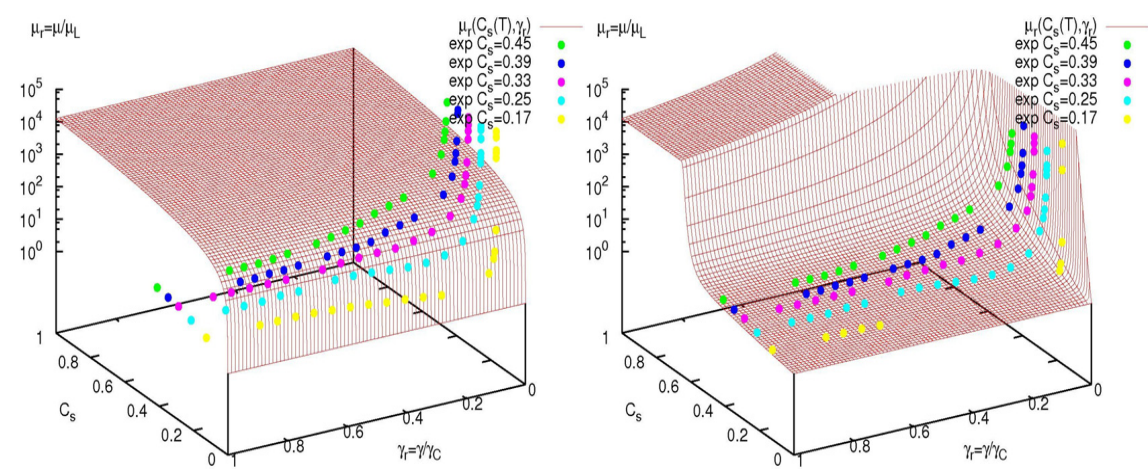

Figure 6: Comparison of the two effective viscosity parametrisations a) linear viscosity see Eq. (13), b) non-linear viscosity cf. Eqs. (16-17).

In Fig. 6 the proposed viscosity parametrisation is compared with experimental data and the old parametrisation given by Eq. (13). One can notice that the chosen approach allows for relatively accurate approximation of the experimental data unlike the original linear approach given by Eq. (13) that over-predicts viscosity values for $C_{S} \leq 0.5$.
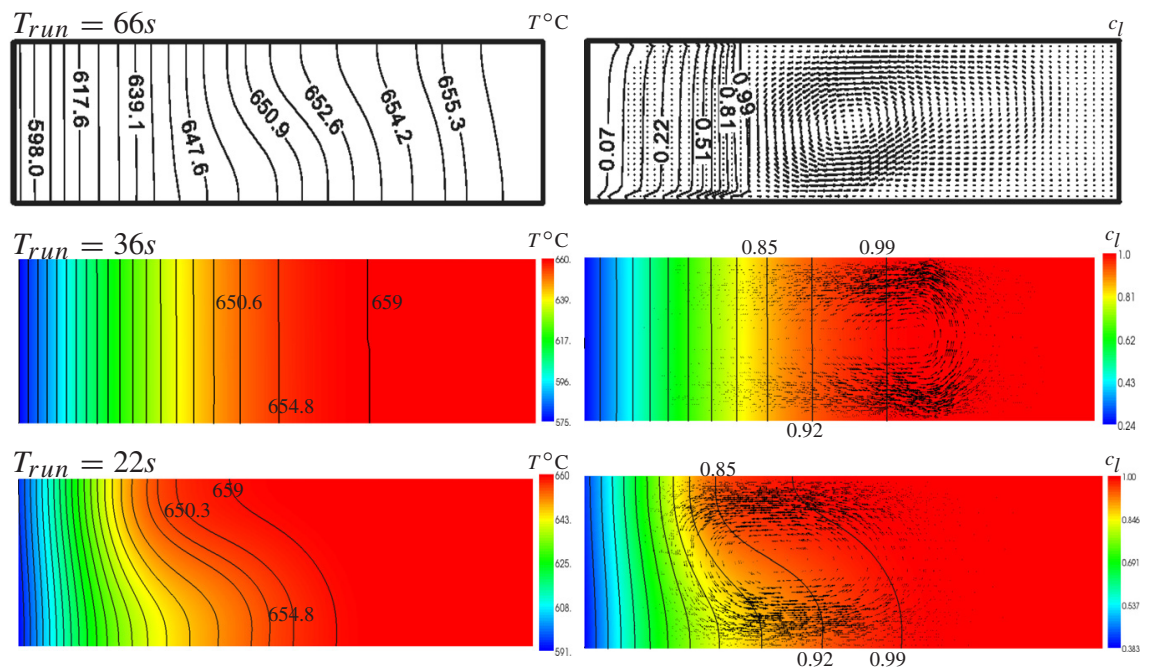

Figure 7: Comparison of the isotherms (left) and the liquid volume fraction isolines (right) in the case of solidification in the rectangular cavity. The two top figures come from the Ref. [3] at $T_{\text {run }}=66 \mathrm{~s}$, the two figures in the middle present the Star-CD result with the linear viscosity model at $T_{\text {run }}=36 \mathrm{~s}$ and the two bottom figures depict the Star-CD solution obtained with the proposed effective viscosity model at $T_{\text {run }}=22 \mathrm{~s}$. 


\section{Solidification of the metal alloy in the rectangular cavity}

In order to access the properties of the proposed effective viscosity parametrisation solidification of an Al-Cu alloy $\left(\mu_{l}=0.003 \mathrm{~Pa} \cdot s, T_{s}=548^{\circ} \mathrm{C}, T_{l}=660^{\circ} \mathrm{C}\right.$ and $\left.\rho=2525 \mathrm{~kg} / \mathrm{m}^{3}\right)$ in a rectangular cavity $(20 \mathrm{~mm} \times 67 \mathrm{~mm})$ is chosen. A detailed description of the test case is given in Ref. [3].

In order to implement the viscosity model given by Eqs. (16-17) an approximation of the error function by elementary function is used (see Ref. [11]) since $\operatorname{erf}()$ is not supplied by the compiler intrinsic functions library. The relative shear velocity $\gamma_{r}$ is approximated by the second invariant of the strain rate tensor that can be considered as the mean shear rate.

Initially, the binary alloy has constant temperature $T_{i}=660^{\circ} \mathrm{C}$ in the whole cavity. The convective boundary condition $q=-h_{\text {conv }}\left(T-T_{a m b}\right)\left(h_{\text {conv }}=\right.$ $1 \mathrm{~kW} /\left(\mathrm{m}^{2 \circ} \mathrm{C}\right), T_{a m b}=20^{\circ} \mathrm{C}$ and $T$ is temperature in the domain) causes a drop of the temperature close to the left side of the cavity and initialise solidification process. The obtained results in Fig. 7 are compared for different run times $T_{\text {run }}$ because it was noticed that due to the Courant number restriction a restart is required with a ten times smaller time step $(\Delta t=1 e-5 s)$. Despite this fact it is possible to compare the main features of the reference solution and the obtained results. From Fig. 7 it is clear that the over prediction of the viscosity by the first model, see also Fig. 6, is responsible for the lack of deformation in the mushy zone visible in the case of the new viscosity parametrisation given by Eqs. (16-17). The temperature distribution and the isotherms obtained with the new parametrisation are closer to the reference solution. As mentioned before, in the case of the solidification model used in Star-CD the front of the solidification always follows isotherms unlike in the reference solution. Moreover, because the first order upwind scheme is the only available discretization for the convective term in Eq. (4), smearing due to the numerical diffusion influences the front of solidification in Fig. 7. Finally, the magnitude of the velocity generated by buoyancy effects is closer to the reference solution in the case of the non-linear viscosity model, however, final quantitative comparison can only be performed when the final result is obtained.

\section{Conclusions}

The paper concerns the binary alloy solidification with the effective viscosity model. It was shown that the effective viscosity assumption can be used in the modelling of the multiphase flow when the solid mass fraction $C_{s} \leq 0.45$ showing good agreement with experimental evidence from cylindrical rheometer. Moreover, the influence of the Taylor instability on the measurements in the cylindrical rheometer was emphasised. The proposed viscosity parametrisation covers more accurately the distribution of the experimental data and gives a realistic solution. Further work on this subject should be devoted to the determination of reliable verification test case based on the experimental evidence. 


\section{References}

[1] Huppert, H.E. \& Worster, M.G., Dynamic solidification of binary melt. Nature, 314, pp. 703-708, 1985.

[2] Peppin, L., Aussillous, P., Huppert, H. \& Worster, G., Steady state mushy layer experiments and theory. J Fluid Mech, 570, pp. 69-77, 2007.

[3] Samanta, D. \& Zabaras, N., Numerical study of macrosegregation in aluminium alloys solidifying on uneven surfaces. Int J Heat Mass Transfer, 48, pp. 4541-4556, 2005.

[4] Nikrityuk, P.A., Eckert, K. \& Grundmann, R., A numerical study of unidirectional solidification of a binary metal alloy under influence of a rotating magnetic field. Int J Heat Mass Transfer, 49, pp. 1501-1515, 2006.

[5] Ni, J. \& Incropera, F.P., Extension of the continuum model for transport phenomena occuring during metall alloy solidification $-i$. the conservation equations. Int J Heat Mass Transfer, 38, pp. 1271-1284, 1995.

[6] Le Bars, M. \& Worster, M.G., Interfacial conditions between a pure fluid and a porous medium: implications for binary alloy solidification. J Fluid Mech, 550, pp. 149-173, 2006.

[7] Roplekar, J.K. \& Dantzing, J.A., A study of solidification with rotating magnetic field. Int J Cast Met Res, 14, pp. 79-98, 2001.

[8] Modigell, M., Pape, L. \& Moll, A., Cylindrical rheometer viscosity measurements of the Al-Si alloy. Aachener Verfahrenstechnik, Personal communication, 2008.

[9] Costa, A., Viscosity of high crystal content melts: depencence on solid fraction. arXiv:physics, 0510191v1, 2005.

[10] Le Bars, M. \& Worster, M.G., Solidification of a binary alloy: Finite-element, single-domain simulation and new benchmark solutions. J Comp Phys, 216, pp. 247-253, 2006.

[11] Error function, approximation with elementary functions. Wikipedia The Free Encyklopedia. 